\title{
Effect of new onset of jaundice on outcomes in patients with sepsis: A population-based propensity score-matched study
}

\section{Desheng Qi}

Xiangya Hospital Central South University

\section{Fuxing Deng}

Xiangya Hospital Central South University

\section{Yuhang Ai}

Xiangya Hospital Central South University

\section{Lina Zhang}

Xiangya Hospital Central South University

\section{Shuangping Zhao}

Xiangya Hospital Central South University

\section{Daomiao Xu}

Xiangya Hospital Central South University

Milin Peng ( $\nabla$ pengmilin@csu.edu.cn )

Xiangya Hospital Central South University https://orcid.org/0000-0002-5443-5646

\section{Research}

Keywords: Sepsis, Jaundice, Liver, Risk factors, Mortality

Posted Date: March 6th, 2020

DOI: https://doi.org/10.21203/rs.3.rs-16270/v1

License: (9) This work is licensed under a Creative Commons Attribution 4.0 International License. Read Full License 


\section{Abstract}

Background Although jaundice have been associated with mortality in critically ill patients, yet no data from large studies demonstrates its effect in sepsis. The aim of the study is to investigate the impact of jaundice on outcomes in septic patients.

Methods Propensity-matched analysis of cohort database from Medical Information Mart for Intensive Care III (MIMIC-III), a large database of septic patients at a tertiary care hospital in Boston, Massachusetts (June 2001 to October 2012). Individuals with preexisting jaundice or liver diseases at the time of admission were excluded from analysis. Jaundice was diagnosed in septic patients with total bilirubin levels $>2 \mathrm{mg} / \mathrm{dL}$ at any time during hospitalization. The multivariate Cox was employed to adjust for baseline and confounding parameters.

Results A total of 2784 septic patients were enrolled in the study, including 456 patients in jaundice group, and 2328 in non-jaundice group. Before propensity score matching, multivariate Cox hazard analysis showed age [HR 1.029; 95\% Cl (1.009-1.049); $\mathrm{P}=0.005$ ], malignancy [HR 3.244; $95 \% \mathrm{Cl}(1.729 \mathrm{~h}-$ 6.085); $P<0.001]$, SOFA score [HR 1.179; $95 \% \mathrm{Cl}(1.054-1.318) ; P=0.004$ ], serum total bilirubin at hospital discharge [HR 1.050; 95\% Cl (1.022-1.079); $\mathrm{P}<0.001]$ were the independent risk factors of mortality in sepsis. In 432 pairs after matching according to new presented jaundice status, jaundice group had higher in-hospital mortality ( $76 \pm 17.6$ vs. $49 \pm 11.3 ; P=0.012)$ than non-jaundice group. In multivariate logistic regression model, the only independent risk for jaundice in sepsis was SOFA score [OR 1.314; $95 \%$ $\mathrm{Cl}$ (1.248-1.385); $\mathrm{P}<0.001$ ], whereas mechanical ventilation [OR 0.310; $95 \% \mathrm{Cl}(0.222-0.432) ; \mathrm{P}<0.001$ ], serum platelet [OR 0.998; $95 \% \mathrm{Cl}(0.997-1.00) ; \mathrm{P}=0.015$ ] and serum bicarbonate [OR $0.962 ; 95 \% \mathrm{Cl}(0.929-$ 0.996); $P=0.030$ ] were jaundice's independent protective factors.

Conclusions New onset of jaundice is associated with higher risk rate of in-hospital mortality in sepsis. In addition, our study demonstrates that serum total bilirubin at hospital discharge is an independent determinant for mortality.

\section{Introduction}

Jaundice raises growing attention and plays an important role in critical disease for decades. There is about $31.2 \%$ incidence rate of jaundice (serum total bilirubin $\geq 2 \mathrm{mg} / \mathrm{dl}$ ) in critical illness (1). Jaundice is associated with poor clinical outcomes in critically ill patients. What' more, the severity of jaundice is closely related to the increasing mortality and the number of failing organs (2). Sepsis is reported to be the main cause of jaundice in critical illness (1). Hyperbilirubinemia occurred in severe illness including sepsis derives from three main causes: hemolysis; hepatic dysfunction and cholestasis. Hepatic dysfunction and intrahepatic cholestasis are regarded as primary causes for jaundice in sepsis (3). Liver acts as the central role in sepsis with functions of bacterial clearance, regulating inflammatory and immune response. Meanwhile liver is a target of sepsis and decompensation of liver function can trigger overwhelming inflammation, immune response and organ damage in sepsis (4-6). Persistent rising 
jaundice may serve as a marker of the host's liver dysfunction in progress of sepsis. And jaundice associated unfavorable prognosis may primarily relate to the extrahepatic complications of the systemic inflammation in sepsis.

Several small scale studies explore the relationship between jaundice and bacteria infection (7), or infant jaundice and sepsis (8), however, yet there are no data from large scale studies investigating the characteristic and influence of jaundice in adults with sepsis. And no study focuses on new onset of jaundice in sepsis before. We use an openly available critical care database, MIMIC-III, to evaluate the risks of new onset of jaundice on clinical outcomes in a large cohort of septic patients.

\section{Methods}

\section{Study design and setting}

We conducted the cohort study according to the STrengthening the Reporting of OBservational studies in Epidemiology (STROBE) statement by using the Medical Information Mart for Intensive Care (MIMIC)-III database, a large, integrated, de-identified, open-free, comprehensive clinical dataset, comprised of all the patients admitted to the ICUs Beth Israel Deaconess Medical Center in Boston, MA, from June 2001 to October 2012. For all of the data are deidentified, patient consent or ethics approval will not be needed. Demographics records, laboratory results, radiology examinations, diagnosis, clinical treatment parameters and dates of death were also concluded. The diagnosed diseases by physician were according to International Classification of Diseases, 9th revision (ICD-9) on patient discharge. Since the study was an analysis of a third-party anonymized publicly available database with preexisting institutional review board (IRB) approval, approval from our institution was exempted.

\section{Participants}

Sepsis was defined according to Sepsis-3 criteria: suspected infection and Sequential Organ Failure Assessment (SOFA) score was of 2 points or more $(9,10)$. Jaundice was diagnosed in patients with new onset of serum total bilirubin $>2 \mathrm{mg} / \mathrm{dL}$ during the stay in the ICU for excluding slight and possible artifactual bilirubin rise under $2 \mathrm{mg} / \mathrm{dL}$ (normal $<1 \mathrm{mg} / \mathrm{dL}$ ). The included criteria were according as follows: age $\geq 18$ years old; patients without previous chronic liver diseases according to the recorded ICD-9 codes, including liver cirrhosis; at ICU admission more than 24 hours; missing data less than $50 \%$. The excluded criteria were including preexisting bilirubin $>2 \mathrm{mg} / \mathrm{dL}$ before hospitalization, and previous chronic liver diseases like chronic hepatitis, acute-on-chronic liver failure, cirrhosis, or acute liver conditions like drug or toxin induced hepatitis. After included in, all the septic patients were divided into jaundice group (serum total bilirubin $>2 \mathrm{mg} / \mathrm{dL}$ ) and non-jaundice group (serum total bilirubin $\leq 2$ $\mathrm{mg} / \mathrm{dL})$.

\section{Measures}


For the patients in the study, we retrieved demographic and admission information from the database during the first $24 \mathrm{~h}$ of ICU admission, including age, gender, ethnicity, time of admission, severity of illness parameters (SOFA score, the Elixhauser comorbidity score), and vital signs (heart rate, and respiratory rate). In addition, we routinely collected laboratory parameters within the first $24 \mathrm{~h}$ of ICU admission. We also collected parameters in the course of sepsis: use of mechanical ventilation, use of vasopressor agents and use of sedative drugs. Total bilirubin (TBIL) was collected in 24h, 48h, 72h, 7d and the day of discharge after ICU admission. We also included several comorbidities according to the recorded ICD-9 codes, including congestive heart failure (CHF), renal disease, atrial fibrillation (AFIB), coronary artery disease (CAD), chronic obstructive pulmonary disease (COPD), stroke, and malignant tumor.

We conducted the follow-up at day 30 (30d), 90d, $180 \mathrm{~d}$ and one-year from the database. The primary outcome was in-hospital mortality, which was defined as the survival status at the time of discharge.

\section{Statistical Analysis}

Statistical analysis was performed using SPSS 23 (SPSS, Inc., Chicago, IL). Baseline characteristics and clinical parameters after ICU admission between jaundice group and control group were compared by using either Student $t$ test or rank-sum test as appropriate. Categorical variables were compared by Chisquare test or Fisher's exact test. Furthermore, univariate and stepwise multivariate Cox hazard analysis was used to analyze risk factors for one-year mortality in septic patients.

To account for selection bias and potential confounding factors between groups in comparison of outcome, we used the propensity score matching (1:1) to balances covariates for those who had jaundice and those who had not $₫ 432$ pairs $\llbracket$. A multivariable logistic regression model with confounding baseline characteristics, including sex, race/ethnicity, preexisting medical conditions: CHF, AFIB, CAD, COPD, stroke, and malignant tumor as covariates, was used to calculate the propensity score for each patient as the predicted probability of jaundice group. One-to-one nearest-neighbor matching without replacement with a caliper width of $0.20 \mathrm{SD}$ was conducted. We evaluated the standardized mean biases, with a difference of less than $10 \%$, which demonstrated a well balance following propensity score matching between the jaundice and non-jaundice groups.

Survival curves were computed and ploted using the Kaplan-Meier method. A stepwise logistic regression model was constructed to explore the independent risk factors of influence on jaundice in sepsis. A $P$ value of $<0.05$ (two-sided) was considered significant.

\section{Results}

Of the 52,963 ICU admissions from MIMIC-III database, 5784 patients fulfilled the definition of sepsis. 3310 participants were identified into our analysis according to the inclusion criteria, afterwards patients with preexisting liver disease $(n=526)$ was excluded from our study. At last $456(16.4 \%)$ patients were consisted of jaundice group, and the remaining 2328 (83.6\%) patients did not develop Jaundice (Fig. 1). 


\section{Patient characteristics before matching.}

Table 1 showed the notable differences in baseline characteristics between jaundice and non-jaundice groups of sepsis before propensity score matching. The jaundice group had a higher prevalence of other race $(20.6 \%$ vs. $16.3 \% ; P=0.029)$, certain preexisting medical comorbidities, including AFIB $(31.8 \%$ vs. $26.7 \% ; P=0.029)$ and malignancy $(28.5 \%$ vs. $21.4 \% ; P=0.001)$, and lower prevalence of COPD $(8.9 \%$ vs. $14.9 \% ; P=0.029)$, stroke (3.5\% vs. $8.4 \% ; P<0.001)$ and renal disease $(14.9 \%$ vs. $21.7 \% ; P=0.001)$ than the non-jaundice group.

There was also significantly higher severity of illness in Jaundice group than non-jaundice group, with higher SOFA score ( $8.4 \pm 4.0$ vs. $5.7 \pm 3.0 ; P<0.001)$, higher heart rate $(112.9 \pm 24.0$ vs. $107.7 \pm 21.3 ; P<0.001)$, respiratory rate $(29.4 \pm 7.2$ vs. $28.6 \pm 6.5 ; P=0.027)$, and also lower systolic pressure ( $144.5 \pm 27.0$ vs. $148.8 \pm 24.9 ; P=0.001)$, diastolic pressure ( $84.5 \pm 19.7$ vs. $87.3 \pm 20.3 ; P=0.009)$. On $24 \mathrm{~h}$ after admission to ICU, jaundice group had higher rate of vasopressor treatment ( 46.7 vs. $39.3 \% ; P=0.004)$, and changed liver function biomarker, as higher AST ( $639.0 \pm 1434.6$ vs. $168.7 \pm 634.9 ; P<0.001)$, ALT $(532.3 \pm 1371.9$ vs. 122.8 $\pm 514.8 ; P<0.001)$,TBIL 24 h (5.3 \pm 5.3 vs. $0.7 \pm 0.5 ; P<0.001)$, TBIL $48 \mathrm{~h}(3.7 \pm 5.0$ vs. $0.3 \pm 0.4 ; P<0.001)$, TBIL $72 \mathrm{~h}(4.4 \pm 4.8$ vs. $0.7 \pm 0.5 ; P<0.001)$, TBIL $7 \mathrm{~d}(5.3 \pm 6.4$ vs. $0.6 \pm 0.4 ; P<0.001)$, TBIL at the day of discharge ( $5.5 \pm 6.5$ vs. $0.7 \pm 0.5 ; P<0.001)$ and lower serum albumin ( $3.0 \pm 0.7$ vs. $3.2 \pm 0.7 ; P<0.001)$. The Elixhauser comorbidity score (5.3 \pm 7.3 vs. $3.8 \pm 7.1 ; P<0.001)$, serum lactate $(4.1 \pm 3.6$ vs. $3.0 \pm 2.5 ; P<0.001)$, and INR $(2.2 \pm 2.2$ vs. $1.7 \pm 1.6 ; P<0.001)$ were also higher in jaundice group. And the jaundice group had lower serum platelet $(215.6 \pm 123.8$ vs. $261.4 \pm 134.6$; $P<0.001)$, bicarbonate $(23.1 \pm 4.5$ vs. $24.5 \pm 4.7$; $P<0.001)$, sodium ( $140.1 \pm 5.0$ vs. $140.9 \pm 5.9 ; P=0.013)$ and arterial $\mathrm{pCO} 2(46.0 \pm 14.9$ vs. $48.1 \pm 16.1$; $P=0.030$ ) than the non-jaundice group.

The overall in-hospital mortality of patients with sepsis was $12.1 \%$. Patients in jaundice group had significantly higher rates of in-hospital mortality $(17.9 \%$ vs. $11.0 \%, P<0.001), 30 \mathrm{~d}$ mortality $(24.0 \%$ vs. $16.6 \%, P<0.001), 90 \mathrm{~d}$ mortality $(28.3 \%$ vs. $19.9 \%, P<0.001), 180 \mathrm{~d}$ mortality $(30.3 \%$ vs. $22.5 \%, P<0.001)$, and one-year mortality after discharge $(32.8 \%$ vs. $25.0 \%, P<0.001)$ than in non-jaundice group, respectively. Kaplan-Meier's analysis also showed that one-year survival rate was significantly lower in jaundice group than in non-jaundice group $(P=0.0149)$ before matching (Fig.2A).

\section{Univariate and stepwise multivariate Cox hazard analysis of risk factors for mortality in sepsis before matching.}

Further, univariate and stepwise multivariate Cox hazard analysis were used to analyze independent risk factors for mortality in sepsis (Table 2). Age [Hazard ratio, HR 1.029; 95\% Cl (1.009-1.049); $P=0.005$ ], preexisting malignancy [HR 3.244; 95\% Cl (1.729-6.085); $P<0.001]$, SOFA score [HR 1.179; 95\% Cl $(1.054-$ 1.318); $P=0.004$ ] and serum total bilirubin at hospital discharge [HR 1.050; 95\% Cl (1.022-1.079); $P<0.001]$ were independent risk factors for one-year mortality before matching by stepwise multivariate Cox hazard analysis.

\section{Propensity score analysis}


Then, we conducted propensity-score matching to controll possible confounders. One-to-one propensityscore matching yielded 432 pairs (Table 3), baseline characteristics for patient were well balanced between the two groups after matching. Standardized biases for all variables were 0.05 or less. In jaundice group after matching, jaundice were still prone to bring more severity of sepsis, with higher SOFA sore $(8.5 \pm 4.0$ vs. $5.6 \pm 3.0 ; P<0.001)$, heart rate $(113.0 \pm 24.1$ vs. $108.3 \pm 21.1 ; P=0.002)$ and respiratory rate ( $29.4 \pm 7.3$ vs. $28.5 \pm 6.3 ; P=0.043)$, lactate ( $4.1 \pm 3.6$ vs. $3.1 \pm 2.6 ; P<0.001)$, serum BUN ( $37.7 \pm 29.8$ vs. $33.3 \pm 23.6 ; P=0.017)$; lower maximum serum platelet ( $214.3 \pm 123.5$ vs. $261.9 \pm 134.7 ; P<0.001)$, and bicarbonate $(23.0 \pm 4.5$ vs. $24.6 \pm 4.5 ; P<0.001)$ on $24 \mathrm{~h}$ admissionthan the matched non-jaundice group. Jaundice group also had significantly higher levels of liver injury related indicators, as AST (649.5 \pm 1434.7 vs. $221.3 \pm 847.0 ; P<0.001)$, ALT (541.2 \pm 1391.9 vs. $146.9 \pm 567.8 ; P<0.001)$, TBIL $24 \mathrm{~h}$ (5.4 \pm 5.4 vs. $0.8 \pm 0.5 ; P<0.001)$, TBIL 48 h ( $5.8 \pm 5.5$ vs. $0.8 \pm 0.5 ; P<0.001)$.

The matched results also showed that jaundice was associated with significantly increased hospital mortality $(17.6 \%$ vs. $11.3 \% P=0.012)$, but not statistically increased in $30 \mathrm{~d}, 90 \mathrm{~d}, 180 \mathrm{~d}$, and one-year mortality in overall population (Table 3, Fig. 2B).

\section{Univariate and multivariate regression analysis to explore independent predictors affecting jaundice in sepsis after matching}

Factors associated with the development of jaundice were entered into multivariate regression analysis, which revealed that mechanical ventilation [OR 0.310; $95 \% \mathrm{Cl}(0.222-0.432) ; P<0.001$ ], serum platelet [OR $0.998 ; 95 \% \mathrm{Cl}(0.997-1.000) ; P=0.015$ ] and serum bicarbonate at $24 \mathrm{~h}$ admission [OR $0.962 ; 95 \% \mathrm{Cl}(0.929$ $0.996) ; P=0.030]$ were the independent protective factors of new presented jaundice, SOFA score [OR $1.314 ; 95 \% \mathrm{Cl}(1.248-1.385) ; P<0.001]$ was the only independent risk factor of new onset of jaundice during course of sepsis (Table 4).

\section{Discussion}

In this large cohort study, we find the incidence rate of new onset of jaundice in sepsis is $16.4 \%$, which is considerably lower than the rates of jaundice in other critical ill patients $(1,2)$. The difference is mainly due to the jaundice in our study is defined as new onset while previous studies includes patients with preexisting chronic liver conditions (7). Our data also concludes that the new onset of jaundice in sepsis can bring significantly higher in-hospital, 30d, 90d,180d, and one-year mortality. Further multivariate Cox hazard analysis shows that serum total bilirubin at hospital discharge, age, preexisting malignancy, and SOFA score are the independent risk factors for mortality of septic patients.

In our study, jaundice group has three times preexistent rate of malignancy than non-jaundice group. What's more, from the above multivariate Cox analysis results, we find malignancy does affect the longterm survival of patients with sepsis, which is consistent of recent research conclusion (11), so generating great bias when analyzing the effect of jaundice on sepsis. Thus, we use propensity score matching analysis to balance baseline characteristics to minimize confounding bias. After matching, new onset of jaundice is associated with significantly increased in-hospital mortality of septic patients. 
Multivariate regression analysis shows SOFA score within $24 \mathrm{~h}$ on admission is the only independent risk factor of new onset of jaundice during course of sepsis, indicating that severity of illness at $24 \mathrm{~h}$ admission may be the main trigger of jaundice in sepsis. Whereas mechanical ventilation, serum platelet, serum bicarbonate are the independent protective factors of new presented jaundice in sepsis.

After propensity score matching, it is important to note that jaundice is associated with a significantly increased risk of in-hospital mortality in sepsis, not in long-term rate of mortality. As we known, the longterm survival rate of sepsis can be affected by preexisting disease, race and other precedent factors, hence, this could explain why there are no statistically significant difference in 30d, 180d, and one-year mortality between jaundice and non-jaundice group after propensity analysis with controlling the confounding covariates.

Furthermore, we try to explore change rule of jaundice in sepsis and try to clarify which stage of jaundice really contributes to mortality of sepsis. Intriguingly, in our results, serum bilirubin level at the day of hospital discharge is the independent risk factor for mortality of septic patients rather than bilirubin level at the time of 24 hour, $48 \mathrm{~h}, 72 \mathrm{~h}$ or 7 day on admission. We can see there is a downtrend at $48 \mathrm{~h}$ for jaundice in septic course, especially apparently in jaundice group, then jaundice level goes up again to near $6 \mathrm{mg} / \mathrm{dl}$ until discharge or demise in jaundice group (Supplemental Fig. 1). In short, our results hints at jaundice may alleviate due to hepatic strong compensation capacity at the beginning of sepsis, liver has the critical regulatory role in sepsis, when liver function becomes decompensated and bilirubin level keeps rising again, this part of patients has higher mortality compared to patients with normal bilirubin level at hospital discharge. Bilirubin variation can serve as a marker of septic change course and bilirubin at the day of hospital discharge can predict endings of sepsis.

In addition, previous study has shown that mechanical ventilation with PEEP $\geq 5 \mathrm{~cm} \mathrm{H}_{2} \mathrm{O}$ is an independent promoting factor for liver dysfunction in critical ill patients (1). A potential link between PEEP and jaundice may be explained as PEEP can decrease cardiac output and portal vein flow and cause an increase in the liver venous resistance leading to ischemia hepatic injury (12). However, we find the use of mechanical ventilation is an independent protective factor for jaundice in sepsis. One of the causes is that we do not record PEEP level of mechanical ventilation and the majority of patients' PEEP level is probably under $5 \mathrm{~cm} \mathrm{H}_{2} \mathrm{O}$ which is not going to aggravate hepatic hypoperfusion. Secondly, hypoxic hepatitis is the main liver injury mechanism during sepsis, which is triggered by inadequate oxgen concentration in the blood $(13,14)$. Hypoxia can cause spasm of small blood vessels and reduce liver blood flow, especially hepatic microcirculation, which can lead to liver damage. Thus, proper early lung protective mechanical ventilation strategy may prevent liver damage through preserving cadiopulmonary function and ameliorating hepatocytes hypoxia. And more studies are needed to investigate the role of mechanical ventilation on liver injury in sepsis.

Of note, the level of platelet significantly decreases in jaundice group compared with the non-jaundice group. And the level of platelet at $24 \mathrm{~h}$ admission is the protective factor of jaundice in our results. Recent studies show that the platelets play a vital role in immunological surveillance against pathogens 
invaders, and contribute to innate immune system function $(15,16)$. Wong and his colleagues demonstrated that platelets collabrate with macrophage to fight against certain blood-borne infections. And the absence of platelet resulted in platelet being unable to localize to the sites of infection, leading to rapid death of the \Kupffer cells and endothelium, following by more leakage of plasma out of blood vessels, and even host mortality (17). Then, the defective bacteria clearance function of liver may result in liver injury, generating hepatogenic jaundice. This may explain why patients with lower level platelet will be more prone to develop jaundice of sepsis in our study. Besides, we find serum bicarbonate is another protective factor of jaundice in sepsis which is consistent with previous experimental studies indicating that endotoxinemia significantly decreases bile acid-independent bile flow (BAIBF) and associated biliary $\mathrm{HCO}_{3}{ }^{-}$output (18), thus reducing serum bicarbonate level. And the exact mechanism of preserved platelet or bicarbonate contributing to hold back jaundice in sepsis needs further study.

To our knowledge, this study is the largest to date investigating the influence of new presented jaundice on outcomes of sepsis. However, there are several limitations in our study. First, we used the database from a single academic medical center in the USA, therefore some of the cases from almost 10 years ago, as diagnosis or treatment strategies at that time would be inconsistent with currently guidelines which brings great bias. And there is residual confounding by variables not collected into MIMIC-III database. However, we include all the septic patients according to the uniform standard of Sepsis 3.0 and apply propensity scores analysis and match the patients' baseline characteristics to eliminate confounding factors and decrease bias to the maximum. Secondly, there are not a few missing data so bringing great bias, yet we delete the data whose missing percentage is larger than $50 \%$ to decrease bias. Third, as SOFA score includes bilirubin grades, there is bias to some extent in our result which shows SOFA score is the indepent risk factor of jaundice in sepsis. Yet SOFA score is a synthetic rating system representing overall severity of critical illness, our result of corelation between SOFA score and jaundice in sepsis has certain referential value. Fourth, the single centered design restricts generalizability to apply our conclusion to other regions. However, single center has some merits for unanimous data from one center can decrease data variation and deviation from different centers when using diverse diagnosis.

\section{Conclusions}

In conclusion, new onset of jaundice is not common finding in sepsis and is associated with an increased risk of in-hospital mortality. Serum total bilirubin at hospital discharge is an independent determinant for one-year mortality. SOFA score, serum platelet, serum bicarbonate at $24 \mathrm{~h}$ on admission, and the use of mechanical ventilation are the independent predictors for new onset of jaundice in sepsis. Our study can raise the awareness of the close corelation between the new onset of jaundice and septic prognosis. Higher quarlity studies with multiple centered design and randomized controlled trials are called for validating and generalizing our conclusion.

\section{Abbreviations}


MIMIC, Medical Information Mart for Intensive Care; TBIL, Total bilirubin; CHF, congestive heart failure; $A F I B$, atrial fibrillation; $C A D$, coronary artery disease; COPD, chronic obstructive pulmonary disease.

\section{Declarations}

\section{Ethics approval and consent to participate}

The establishment of the database was approved by the Massachusetts Institute of Technology (Cambridge, MA) and the Institutional Review Boards of Beth Israel Deaconess Medical Center (Boston, $\mathrm{MA})$.

\section{Consent for publication}

Not applicable.

\section{Availability of data and materials}

The MIMIC III database is available from https:// mimic.physionet.org/.

Competing interests: The authors declare that they have no competing interests.

\section{Funding}

Not applicable.

\section{Authors' contributions}

Peng ML contributed to conceptualization, software, formal analysis, supervision, validation, investigation, visualization, methodology, and writing-review \& editing the research; Qi DS and Deng FX contributed to the data curation and resource; Ai YH, Zhang LN, Zhao SP, and Xu DM contributed to supervision and validation of the data; Qi DS contributed to investigation, writing-original draft and project administration of the manuscript. All authors critically revised the manuscript, agree to be fully accountable for ensuring the integrity and accuracy of the work, and read and approved the final manuscript.

Acknowledgments: We are grateful for professional Professor Liu WW for kind help for consultation of statistical problems.

\section{References}

1. Brienza N, Dalfino L, Cinnella G, Diele C, Bruno F, Fiore T. Jaundice in critical illness: promoting factors of a concealed reality. Intensive Care Med. 2006;32(2):267-74.

2. te Boekhorst T, Urlus M, Doesburg W, Yap SH, Goris RJ. Etiologic factors of jaundice in severely ill patients. A retrospective study in patients admitted to an intensive care unit with severe trauma or 
with septic intra-abdominal complications following surgery and without evidence of bile duct obstruction. J Hepatol. 1988;7(1):111-7.

3. Chand N, Sanyal AJ. Sepsis-induced cholestasis. Hepatology. 2007;45(1):230-41.

4. Yan J, Li S, Li S. The role of the liver in sepsis. Int Rev Immunol. 2014;33(6):498-510.

5. Strnad P, Tacke F, Koch A, Trautwein C. Liver - guardian, modifier and target of sepsis. Nat Rev Gastroenterol Hepatol. 2017;14(1):55-66.

6. Bauer M, Press AT, Trauner M. The liver in sepsis: patterns of response and injury. Curr Opin Crit Care. 2013;19(2):123-7.

7. Franson TR, Hierholzer WJ, Jr., LaBrecque DR. Frequency and characteristics of hyperbilirubinemia associated with bacteremia. Rev Infect Dis. 1985;7(1):1-9.

8. Garcia-Munoz F, Santana C, Reyes D, Wiehoff A, Lopez-Pinto JM, Garcia-Alix A. Early sepsis, obstructive jaundice and right-sided diaphragmatic hernia in the newborn. Acta Paediatr. 2001;90(1):96-8.

9. Singer M, Deutschman CS, Seymour CW, Shankar-Hari M, Annane D, Bauer M, et al. The Third International Consensus Definitions for Sepsis and Septic Shock (Sepsis-3). JAMA. 2016;315(8):80110.

10. Vincent JL, Moreno R, Takala J, Willatts S, De Mendonca A, Bruining H, et al. The SOFA (Sepsisrelated Organ Failure Assessment) score to describe organ dysfunction/failure. On behalf of the Working Group on Sepsis-Related Problems of the European Society of Intensive Care Medicine. Intensive Care Med. 1996;22(7):707-10.

11. Dimopoulos G, Rovina N, Patrani M, Antoniadou E, Konstantonis D, Vryza K, et al. Past history of stage I/II solid tumor malignancy impacts considerably on sepsis mortality: a propensity score matching analysis from the hellenic sepsis study group. BMC Infect Dis. 2019;19(1):831.

12. Brienza N, Revelly JP, Ayuse T, Robotham JL. Effects of PEEP on liver arterial and venous blood flows. Am J Respir Crit Care Med. 1995;152(2):504-10.

13. Spapen H. Liver perfusion in sepsis, septic shock, and multiorgan failure. Anat Rec (Hoboken). 2008;291(6):714-20.

14. Lescot T, Karvellas $C$, Beaussier M, Magder $S$. Acquired liver injury in the intensive care unit. Anesthesiology. 2012;117(4):898-904.

15. Surewaard BGJ, Thanabalasuriar A, Zeng Z, Tkaczyk C, Cohen TS, Bardoel BW, et al. alpha-Toxin Induces Platelet Aggregation and Liver Injury during Staphylococcus aureus Sepsis. Cell Host Microbe. 2018;24(2):271-84 e3.

16. Broadley SP, Plaumann A, Coletti R, Lehmann C, Wanisch A, Seidlmeier A, et al. Dual-Track Clearance of Circulating Bacteria Balances Rapid Restoration of Blood Sterility with Induction of Adaptive Immunity. Cell Host Microbe. 2016;20(1):36-48.

17. Wong CH, Jenne CN, Petri B, Chrobok NL, Kubes P. Nucleation of platelets with blood-borne pathogens on Kupffer cells precedes other innate immunity and contributes to bacterial clearance. 
Nat Immunol. 2013;14(8):785-92.

18. Trauner M, Nathanson MH, Rydberg SA, Koeppel TA, Gartung C, Sessa WC, et al. Endotoxin impairs biliary glutathione and $\mathrm{HCO}-$ excretion and blocks the choleretic effect of nitric oxide in rat liver. Hepatology. 1997;25(5):1184-91.

\section{Tables}

Table1. Demographic characteristics and clinical outcomes between jaundice and non-jaundice groups before propensity score matching. 


\begin{tabular}{|c|c|c|c|}
\hline \multirow[t]{3}{*}{ Parameters } & \multicolumn{2}{|l|}{ Full Cohort } & \multirow[b]{3}{*}{$P$} \\
\hline & \multicolumn{2}{|c|}{ Jaundice group } & \\
\hline & $(\mathrm{n}=456)$ & No-jaundice group $(n=2328)$ & \\
\hline Age, median (SD),y & 65.6(18.3) & $65.5(17.8)$ & 0.933 \\
\hline Male, n(\%) & $259(56.8)$ & $1258(54.0)$ & 0.281 \\
\hline \multicolumn{4}{|l|}{ Race } \\
\hline White & $315(69.1)$ & 1635 (70.2) & 0.615 \\
\hline Hispanic & $13(2.9)$ & $69(3.0)$ & 1.000 \\
\hline Black & $34(7.5)$ & $244(10.5)$ & 0.050 \\
\hline Other & $94(20.6)$ & $380(16.3)$ & 0.029 \\
\hline Weight, median (SD) & $82.6(34.3)$ & $81.4(24.9)$ & 0.585 \\
\hline Height, median (SD) & 169.4(10.1) & $169.0(10.7)$ & 0.417 \\
\hline \multicolumn{4}{|l|}{ Preexisting medical conditions, n(\%) } \\
\hline $\mathrm{CHF}$ & 105(23.0) & $608(26.1)$ & 0.177 \\
\hline AFIB & 145(31.8) & $622(26.7)$ & 0.029 \\
\hline COPD & $41(8.9)$ & $346(14.9)$ & 0.001 \\
\hline $\mathrm{CAD}$ & 93(20.4) & $504(21.6)$ & 0.575 \\
\hline Stroke & $16(3.5)$ & $197(8.4)$ & $<0.001$ \\
\hline Malignancy & $130(28.5)$ & 498(21.4) & 0.001 \\
\hline Renal disease & $68(14.9)$ & $505(21.7)$ & 0.001 \\
\hline SOFA at admission, median (SD) & $8.4(4.0)$ & $5.7(3.0)$ & $<0.001$ \\
\hline Elixhauser comorbidity index, median (SD) & $5.3(7.3)$ & $3.8(7.1)$ & $<0.001$ \\
\hline Vasopressor, n (\%) & $213(46.7)$ & $916(39.3)$ & 0.004 \\
\hline Mechanical ventilation, n (\%) & $228(50.0)$ & $1253(53.8)$ & 0.137 \\
\hline Sedative drug usage & $226(49.6)$ & $1188(51.0)$ & 0.574 \\
\hline Maximum heart rate, median (SD) & $112.9(24.0)$ & $107.7(21.3)$ & $<0.001$ \\
\hline Maximum systolic pressure, median (SD) & $144.5(27.0)$ & $148.8(24.9)$ & 0.001 \\
\hline Maximum diastolic pressure, median (SD) & 84.5(19.7) & $87.3(20.3)$ & 0.009 \\
\hline Maximum respiratory rate, median (SD) & $29.4(7.2)$ & $28.6(6.5)$ & 0.027 \\
\hline \multicolumn{4}{|c|}{ Laboratory parameters within the first $24 \mathrm{~h}$ of ICU admission } \\
\hline Maximum WBC, median (SD) & $16.0(9.3)$ & $16.1(17.9)$ & 0.856 \\
\hline Maximum hemoglobin, median (SD) & $12.1(2.1)$ & $11.9(2.2)$ & 0.252 \\
\hline Maximum platelet, median (SD) & $215.6(123.8)$ & $261.4(134.6)$ & $<0.001$ \\
\hline Maximum potassium, median (SD) & $4.7(0.9)$ & $4.8(1.0)$ & 0.198 \\
\hline Maximum sodium, median (SD) & $140.1(5.0)$ & $140.9(5.9)$ & 0.013 \\
\hline Maximum bicarbonate, median (SD) & $23.1(4.5)$ & $24.5(4.7)$ & $<0.001$ \\
\hline Maximum chloride, median (SD) & $108.0(6.7)$ & $108.3(7.2)$ & 0.521 \\
\hline Maximum Bun, median (SD) & $37.4(29.2)$ & $35.0(27.5)$ & 0.093 \\
\hline Maximum lactate, median (SD) & $4.1(3.6)$ & $3.0(2.5)$ & $<0.001$ \\
\hline Maximum creatinine, median (SD) & $1.9(1.5)$ & $2.0(2.1)$ & 0.751 \\
\hline Maximum hematocrit, median (SD) & $36.1(6.4)$ & $36.0(6.5)$ & 0.756 \\
\hline Maximum INR, median (SD) & $2.2(2.2)$ & $1.7(1.6)$ & $<0.001$ \\
\hline Maximum PH, median (SD) & $7.4(0.1)$ & $7.4(0.1)$ & 0.144 \\
\hline Maximum pO2, median (SD) & $205.9(123.5)$ & 215.6(135.3) & 0.198 \\
\hline Maximum pCO2, median (SD) & $46.0(14.9)$ & $48.1(16.1)$ & 0.030 \\
\hline
\end{tabular}




\begin{tabular}{|c|c|c|c|}
\hline Maximum $\mathrm{CK}$, median (SD) & $1274.1(3587.9)$ & 1209.6(4606.9) & 0.837 \\
\hline Maximum albumin, median (SD) & $3.0(0.7)$ & $3.2(0.7)$ & $<0.001$ \\
\hline Maximum ALT, median (SD) & $532.3(1371.9)$ & $122.8(514.8)$ & $<0.001$ \\
\hline Maximum AST, median (SD) & $639.0(1434.6)$ & 168.7(634.9) & $<0.001$ \\
\hline Maximum total bilirubin at $24 \mathrm{~h}$, median (SD) & $5.3(5.3)$ & $0.7(0.5)$ & $<0.001$ \\
\hline Maximum total bilirubin at $48 \mathrm{~h}$, median (SD) & $3.7(5.0)$ & $0.3(0.4)$ & $<0.001$ \\
\hline Maximum total bilirubin at $72 \mathrm{~h}$, median (SD) & $4.4(4.8)$ & $0.7(0.5)$ & $<0.001$ \\
\hline Maximum total bilirubin at $7 d$, median (SD) & $5.3(6.4)$ & $0.6(0.4)$ & $<0.001$ \\
\hline Maximum total bilirubin at the day of discharge, median (SD) & $5.5(6.5)$ & $0.7(0.5)$ & $<0.001$ \\
\hline The length of hospital days & $5.3(7.1)$ & $4.8(6.0)$ & 0.262 \\
\hline In-hospital mortality, n(\%) & 79 (17.9) & $249(11.0)$ & $<0.001$ \\
\hline 30d mortality, n(\%) & $106(24.0)$ & $376(16.6)$ & $<0.001$ \\
\hline 90d mortality, $\mathrm{n}(\%)$ & $125(28.3)$ & $450(19.9)$ & $<0.001$ \\
\hline 180d mortality, $\mathrm{n}(\%)$ & $134(30.3)$ & $509(22.5)$ & 0.001 \\
\hline One-year mortality, n(\%) & $145(32.8)$ & $565(25.0)$ & 0.001 \\
\hline
\end{tabular}

Continuous variables are reported as mean (Standard deviation, SD), and Categorical variables are reported as count (\% of column total).

Abbreviations: CHF congestive heart failure, AFIB atrial fibrillation, CAD coronary artery disease, COPD chronic obstructive pulmonary disease, SOFA Sequential Organ Failure Assessment, WBC white blood cell, INR International Normalized Ratio, BUN blood urea nitrogen, AST aspartate transaminase, $\mathrm{ALT}$ alanine transaminase, $\mathrm{pCO}_{2}$ partial pressure of carbon dioxide, $\mathrm{pO}_{2}$ partial pressure of oxygen.

Table 2. Univariate and stepwise multivariate Cox hazard analysis of risk factors for mortality in sepsis before matching. 


\begin{tabular}{|c|c|c|c|c|c|c|c|}
\hline \multirow{3}{*}{$\begin{array}{l}\text { Parameters } \\
\text { Age }\end{array}$} & \multicolumn{3}{|c|}{ Univariate analysis } & \multicolumn{4}{|c|}{ Multivariate analysis } \\
\hline & \multirow{2}{*}{$\frac{\mathrm{HR}}{1.028}$} & \multicolumn{2}{|c|}{$95 \% \mathrm{CI}$} & \multirow{2}{*}{$\frac{\mathrm{HR}}{1.029}$} & \multirow{2}{*}{$\frac{95 \% \text { CI }}{1.009-1.049}$} & \multirow{2}{*}{\multicolumn{2}{|c|}{$\frac{P}{0.005}$}} \\
\hline & & $1.023-1.032$ & $<0.001$ & & & & \\
\hline \multicolumn{8}{|l|}{ Race } \\
\hline Race Hispanic & 0.437 & $0.234-0.816$ & 0.009 & - & & & \\
\hline Race other & 1.412 & $1.181-1.688$ & $<0.001$ & - & & & \\
\hline \multicolumn{8}{|l|}{ Preexisting medical conditions } \\
\hline Malignancy & 2.211 & $1.898-2.577$ & $<0.001$ & 3.244 & $1.729-6.085$ & $<0.001$ & \\
\hline Stroke & 1.524 & $1.200-1.936$ & 0.001 & - & & & \\
\hline AFIB & 1.520 & $1.303-1.773$ & $<0.001$ & - & & & \\
\hline Respiratory rate & 1.020 & $1.010-1.031$ & $<0.001$ & - & & & \\
\hline Heart rate & 1.005 & $1.002-1.009$ & 0.002 & - & & & \\
\hline SOFA & 1.115 & 1.094-1.136 & $<0.001$ & 1.179 & $1.054-1.318$ & 0.004 & \\
\hline The use of vasoactive drug & 1.447 & $1.248-1.676$ & $<0.001$ & 0.470 & $0.214-1.029$ & 0.059 & \\
\hline Acute kidney failure at $24 \mathrm{~h}$ admission & 1.265 & $1.189-1.347$ & $<0.001$ & - & & & \\
\hline Sedative usage & 1.065 & $0.919-1.234$ & 0.399 & - & & & \\
\hline Mechanical ventilation & 1.261 & $1.086-1.464$ & 0.002 & 0.548 & $0.268-1.120$ & 0.099 & \\
\hline \multicolumn{8}{|c|}{ Laboratory parameters within the first $24 \mathrm{~h}$ of ICU admission } \\
\hline INR & 1.057 & $1.025-1.091$ & 0.001 & - & & & \\
\hline Lactate & 1.090 & $1.070-1.111$ & $<0.001$ & - & & & \\
\hline Bicarbonate & 0.970 & 0.954-0.986 & $<0.001$ & - & & & \\
\hline Maximum potassium & 1.125 & $1.052-1.202$ & 0.001 & - & & & \\
\hline BUN & 1.007 & $1.005-1.009$ & $<0.001$ & - & & & \\
\hline Creatinine & 1.014 & $0.981-1.048$ & 0.415 & - & & & \\
\hline The occurrence of jaundice & 1.388 & $1.157-1.666$ & $<0.001$ & - & & & \\
\hline Serum total bilirubin at hospital $24 \mathrm{~h}$ & 1.039 & $1.021-1.057$ & $<0.001$ & - & & & \\
\hline Serum total bilirubin at hospital $48 \mathrm{~h}$ & 1.043 & $1.024-1.063$ & $<0.001$ & - & & & \\
\hline Serum total bilirubin at hospital $72 \mathrm{~h}$ & 1.046 & $1.025-1.068$ & $<0.001$ & - & & & \\
\hline Serum total bilirubin at hospital $7 \mathrm{~d}$ & 1.050 & $1.026-1.074$ & $<0.001$ & - & & & \\
\hline Serum total bilirubin at discharge & 1.044 & $1.027-1.062$ & $<0.001$ & 1.050 & $1.022-1$ & 079 & $<0.001$ \\
\hline
\end{tabular}


Table3. Demographic characteristics and clinical outcomes for jaundice and non-jaundice groups after propensity score matching.

Page 15/20 


\begin{tabular}{|c|c|c|c|}
\hline \multirow[t]{3}{*}{ Parameters } & \multicolumn{2}{|c|}{ Propensity Score-Matched Cohort } & \multirow[b]{3}{*}{$P$} \\
\hline & \multicolumn{2}{|l|}{ Jaundice group } & \\
\hline & $(\mathrm{n}=432)$ & No-jaundice group $(\mathrm{n}=432)$ & \\
\hline Age, median (SD),y & $65.1(18.3)$ & $65.6(17.3)$ & 0.702 \\
\hline Male, $n(\%)$ & $249(57.6)$ & $231(53.5)$ & 0.244 \\
\hline \multicolumn{4}{|l|}{ Race } \\
\hline White & $297(68.8)$ & $303(70.1)$ & 0.712 \\
\hline Hispanic & 13(3.0) & $9(2.0)$ & 0.518 \\
\hline Black & 29(6.7) & $27(6.3)$ & 0.890 \\
\hline Other & 93 (21.5) & 93 (21.5) & 1.000 \\
\hline \multicolumn{4}{|l|}{ Preexisting medical conditions, n(\%) } \\
\hline $\mathrm{CHF}$ & $95(22.0)$ & $111(25.7)$ & 0.231 \\
\hline AFIB & 135(31.3) & $121(28.0)$ & 0.333 \\
\hline COPD & $39(9.0)$ & $35(8.1)$ & 0.716 \\
\hline $\mathrm{CAD}$ & $88(20.4)$ & 93(21.5) & 0.738 \\
\hline Stroke & 14(3.2) & 17(3.9) & 0.715 \\
\hline Malignancy & $120(27.8)$ & 115(26.6) & 0.760 \\
\hline Renal disease & $64(14.8)$ & $82(19.0)$ & 0.123 \\
\hline SOFA at admission, median (SD) & $8.5(4.0)$ & $5.6(3.0)$ & $<0.001$ \\
\hline Elixhauser comorbidity index, median (SD) & $5.2(7.4)$ & $4.5(7.3)$ & 0.139 \\
\hline Vasopressor, n (\%) & 203(47.0) & $178(41.2)$ & 0.100 \\
\hline Mechanical ventilation, n (\%) & 215(49.8) & $233(53.9)$ & 0.247 \\
\hline Maximum heart rate, median (SD) & $113.0(24.1)$ & 108.3(21.1) & 0.002 \\
\hline Maximum systolic pressure, median (SD) & 144.4(27.5) & 146.2(23.0) & 0.303 \\
\hline Maximum diastolic pressure, median (SD) & $84.4(19.5)$ & $86.2(19.5)$ & 0.186 \\
\hline Maximum respiratory rate, median (SD) & 29.4(7.3) & 28.5(6.3) & 0.043 \\
\hline \multicolumn{4}{|c|}{ Laboratory parameters within the first $24 \mathrm{~h}$ of ICU admission } \\
\hline Maximum WBC, median (SD) & $16.1(9.4)$ & 18.2(28.7) & 0.856 \\
\hline Maximum hemoglobin, median (SD) & $12.1(2.2)$ & $12.0(2.2)$ & 0.252 \\
\hline Maximum platelet, median (SD) & 214.3(123.5) & $261.9(134.7)$ & $<0.001$ \\
\hline Maximum potassium, median (SD) & $4.7(0.9)$ & $4.7(1.0)$ & 0.486 \\
\hline Maximum sodium, median (SD) & $140.1(5.1)$ & $140.8(5.7)$ & 0.054 \\
\hline Maximum bicarbonate, median (SD) & $23.0(4.5)$ & $24.6(4.5)$ & $<0.001$ \\
\hline Maximum chloride, median (SD) & $108.0(6.7)$ & 108.3(6.8) & 0.556 \\
\hline Maximum Bun, median (SD) & $37.7(29.8)$ & $33.3(23.6)$ & 0.017 \\
\hline Maximum lactate, median (SD) & $4.1(3.6)$ & $3.1(2.6)$ & $<0.001$ \\
\hline Maximum creatinine, median (SD) & $2.0(1.5)$ & $1.9(1.7)$ & 0.463 \\
\hline Maximum hematocrit, median (SD) & $36.1(6.5)$ & $36.2(6.5)$ & 0.846 \\
\hline Maximum ALT, median (SD) & $541.2(1391.9)$ & 146.9(567.8) & $<0.001$ \\
\hline Maximum AST, median (SD) & $649.5(1434.7)$ & 221.3(847.0) & $<0.001$ \\
\hline Maximum bilirubin at 24h, median (SD) & $5.4(5.4)$ & $0.8(0.5)$ & $<0.001$ \\
\hline Maximum bilirubin at $48 \mathrm{~h}$, median (SD) & $5.8(5.5)$ & $0.8(0.5)$ & $<0.001$ \\
\hline In-hospital mortality, n(\%) & 76 (17.6) & 49 (11.3) & 0.012 \\
\hline 30d mortality, n(\%) & $102(23.6)$ & $82(19.0)$ & 0.114 \\
\hline 90d mortality, n(\%) & $121(28.0)$ & $102(23.6)$ & 0.162 \\
\hline
\end{tabular}


Continuous variables are reported as mean (Standard deviation, SD), and Categorical variables are reported as count (\% of column total).

Abbreviations: CHF congestive heart failure, AFIB atrial fibrillation, CAD coronary artery disease, COPD chronic obstructive pulmonary disease, SOFA Sequential Organ Failure Assessment, WBC white blood cell, INR International Normalized Ratio, BUN blood urea nitrogen, AST aspartate transaminase, ALT alanine transaminase, $\mathrm{pCO} 2$ partial pressure of carbon dioxide, pO2 partial pressure of oxygen.

Table 4. Univariate and multivariate regression analysis to explore independent predictors affecting jaundice in sepsis

\begin{tabular}{|c|c|c|c|c|c|c|}
\hline \multirow[t]{2}{*}{ Parameters } & \multicolumn{2}{|c|}{ Univariate } & \multicolumn{3}{|c|}{ Multivariate analysis } & \multirow[b]{2}{*}{$P$} \\
\hline & OR & $95 \% \mathrm{CI}$ & $P$ & OR & $95 \% \mathrm{CI}$ & \\
\hline Mechanical ventilation & 0.668 & $0.526-0.900$ & 0.006 & 0.310 & $0.222-0.432$ & $<0.001$ \\
\hline Vasoactive drug usage & 1.172 & $0.897-1.533$ & 0.245 & - & & \\
\hline SOFA & 1.246 & 1.192-1.302 & $<0.001$ & 1.314 & $1.248-1.385$ & $<0.001$ \\
\hline Platelet at 24 admission & 0.997 & 0.996-0.998 & $<0.001$ & 0.998 & $0.997-1.000$ & 0.015 \\
\hline Bicarbonate at 24 admission & 0.934 & 0.906-0962 & $<0.001$ & 0.962 & 0.929-0.996 & 0.030 \\
\hline Lactate at $24 \mathrm{~h}$ admission & 1.121 & 1.069-1.176 & $<0.001$ & - & & \\
\hline Heart rate & 1.012 & 1.006-1.018 & $<0.001$ & 1.006 & 0.999-1.012 & 0.095 \\
\hline Respiratory rate & 1.028 & $1.007-1.048$ & 0.008 & - & & \\
\hline
\end{tabular}

Abbreviations: SOFA Sequential Organ Failure Assessment.

\section{Supporting Information}

Supplemental Fig. 1. Trend chart of serum total jaundice level in the course of sepsis in jaundice group and non-jaundice group before matching.

\section{Figures}




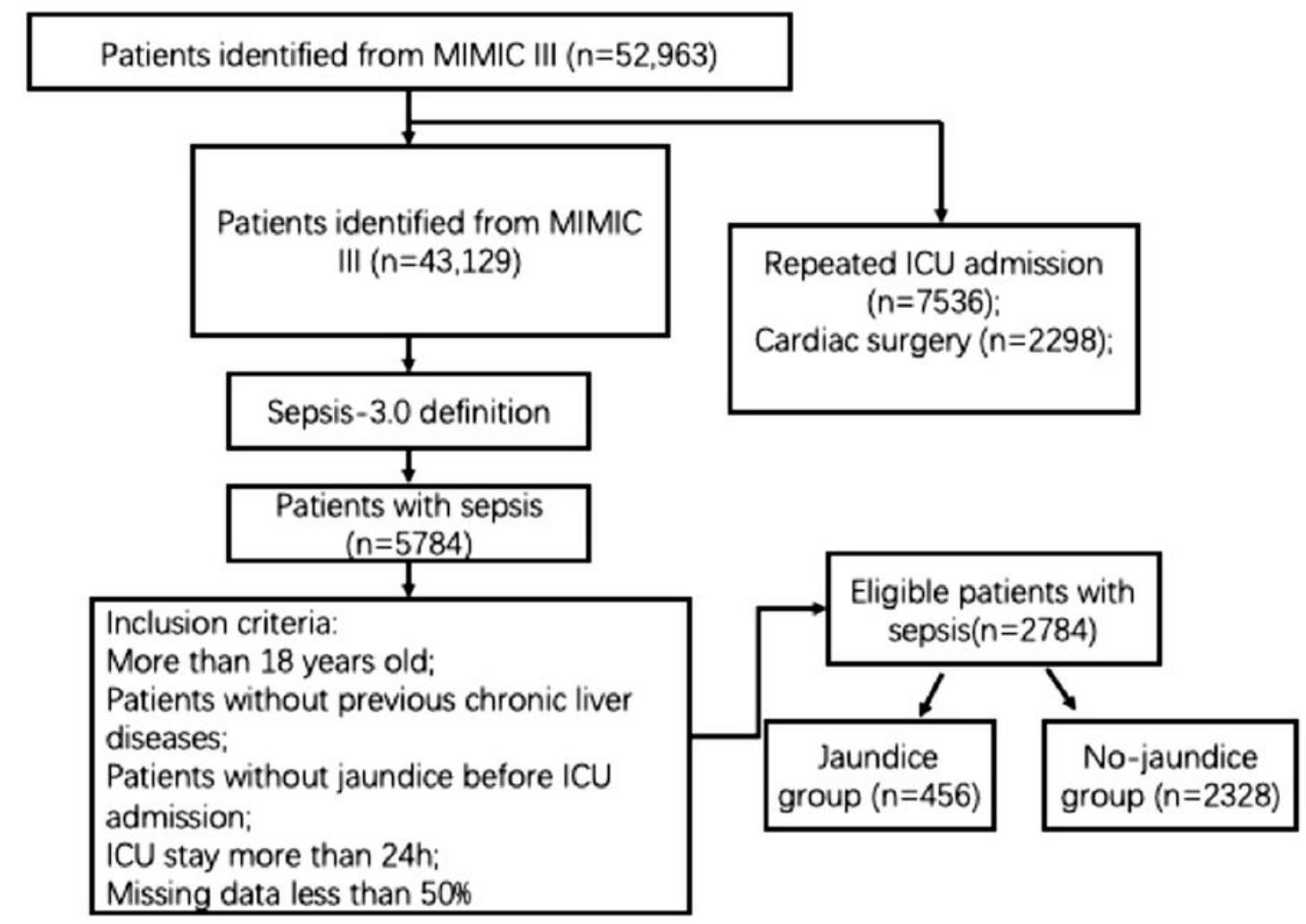

\section{Figure 1}

Flowchart of patient selection. 
A

- No-Jaundice group

- Jaundice group

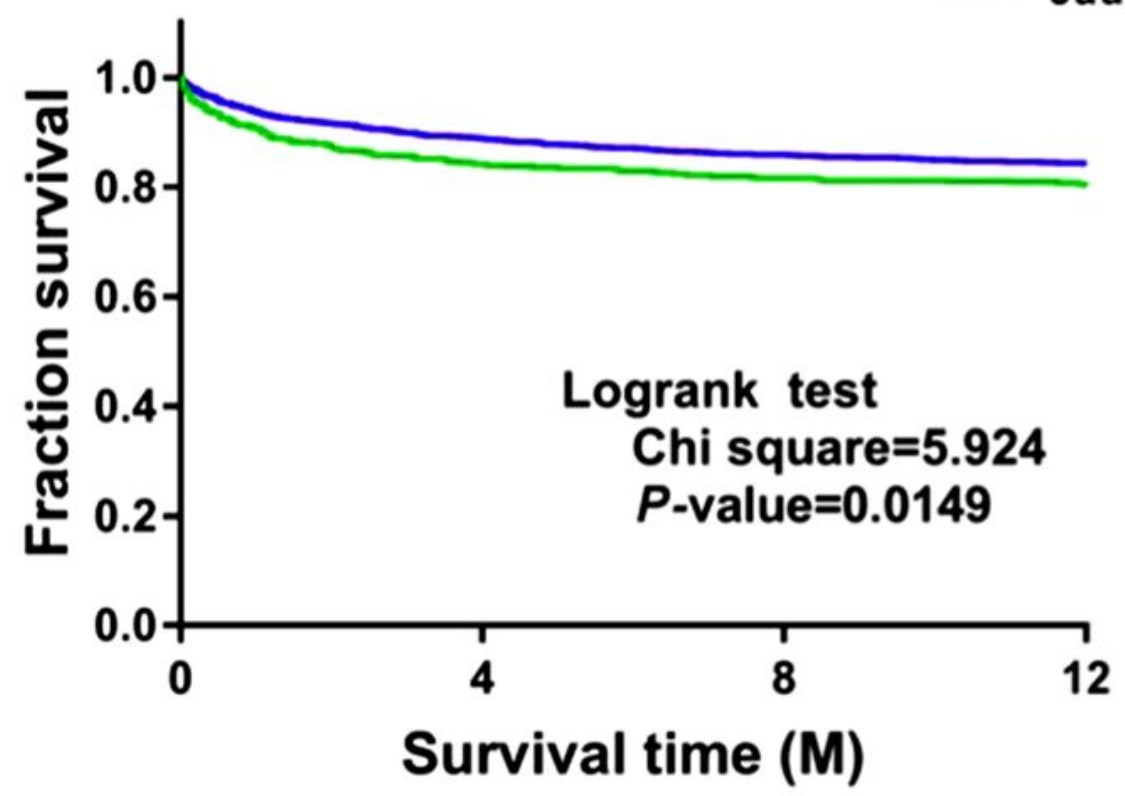

B

- No-Jaundice group

- Jaundice group

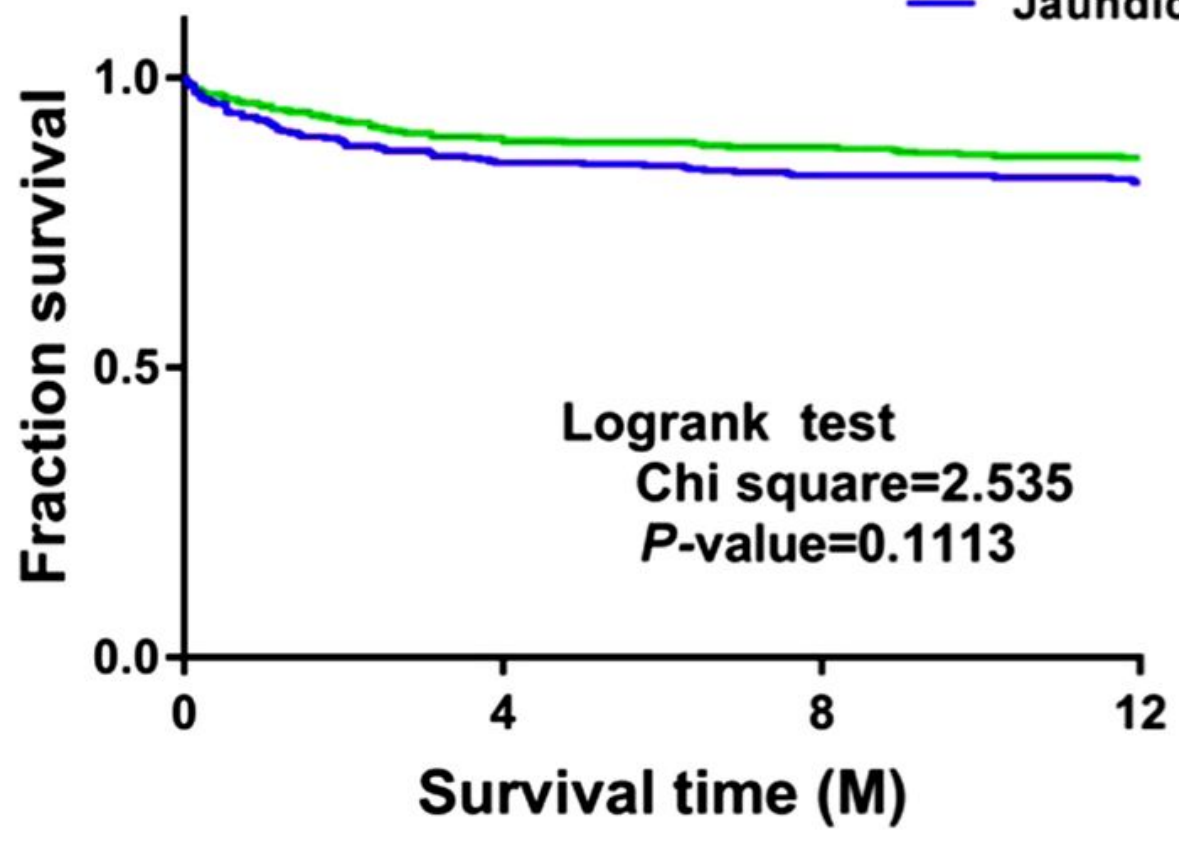

Figure 4

Time to death analysis. A. shows the survival curves for all included patients at one year after randomization of the last patients before matching, with significant different between two groups (Log rank $\mathrm{P}=0.0149$ ); $\mathrm{B}$. shows the survival curves for propensity matched patients at one year after randomization of the last patients. Kaplan-Meier analysis showed survival time did not differ between the jaundice group and the normal group. Log rank $\mathrm{P}=0.1113$. 


\section{Supplementary Files}

This is a list of supplementary files associated with this preprint. Click to download.

- SFig1.tif

- SFig1.tif 